Revue d'histoire de l'Amérique française

DEVUE D.HISTOIRE DE L'AMÉRIQUE FRANÇAISE

\title{
Ville et santé au Québec un bilan de la recherche historique
}

\section{François Guérard}

Volume 53, numéro 1, été 1999

Médecine, santé et sociétés

URI : https://id.erudit.org/iderudit/005459ar

DOI : https://doi.org/10.7202/005459ar

Aller au sommaire du numéro

Éditeur(s)

Institut d'histoire de l'Amérique française

ISSN

0035-2357 (imprimé)

1492-1383 (numérique)

Découvrir la revue

Citer cet article

Guérard, F. (1999). Ville et santé au Québec un bilan de la recherche historique. Revue d'histoire de l'Amérique française, 53(1), 19-45.

https://doi.org/10.7202/005459ar

\section{Résumé de l'article}

La santé en milieu urbain au Québec n'est devenue objet d'étude pour les historiens qu'à partir de la décennie 1970. Depuis, divers chercheurs qui inscrivaient leur démarche dans le courant de l'histoire sociale ont réexaminé, du point de vue de la santé, la ville et sa population, ses institutions et ses services. Dans quelles perspectives les ont-ils abordés? Comment les ont-ils intégrés à leurs problématiques? Quelle a été leur contribution à la compréhension de la société québécoise? Un bilan historiographique est présenté qui cherche à répondre à ces questions, tout en mettant en perspective les acquis de recherche et les avenues encore négligées. 


\title{
VILLE ET SANTÉ AU QUÉBEC UN BILAN DE LA RECHERCHE HISTORIQUE ${ }^{1}$
}

\author{
FRANÇOIS GUÉRARD \\ Centre interuniversitaire d'études québécoises \\ Université du Québec à Trois-Rivières
}

\section{RÉSUMÉ}

La santé en milieu urbain au Québec n'est devenue objet d'étude pour les historiens qu'à partir de la décennie 1970. Depuis, divers chercheurs qui inscrivaient leur démarche dans le courant de l'histoire sociale ont réexaminé, du point de vue de la santé, la ville et sa population, ses institutions et ses services. Dans quelles perspectives les ont-ils abordés? Comment les ont-ils intégrés à leurs problématiques? Quelle a été leur contribution à la compréhension de la société québécoise? Un bilan historiographique est présenté qui cherche à répondre à ces questions, tout en mettant en perspective les acquis de recherche et les avenues encore négligées.

\section{ABSTRACT}

Quebec historians' interest in urban health is a recent phenomenon, originating in the 1970s. Since then, researchers drawing on the methods and concerns of social history have asked new questions bearing on the health of the city's population and on its relevant institutions and services. The following article reviews this recent literature, delineating its findings, approaches, and perspectives, and outlines various areas that have as yet been neglected.

Depuis une vingtaine d'années, l'histoire de la santé et de la médecine au Québec s'est considérablement développée, alors que les publications de travaux savants se succédaient à un rythme toujours accéléré. À la fin des années 1970, tout, ou presque, paraissait à faire : l'état de santé des populations et ses déterminants, les services sanitaires et leurs réformes, les pratiques, populaires aussi bien que médicales, les perceptions et les discours, les rapports entre les acteurs sociaux et leurs rôles respectifs, autant de thématiques qui étaient à explorer. L'attrait des nouveaux territoires a joué : nombre d'historiens s'y sont investis.

1. Nous remercions chaleureusement Yvan Rousseau pour ses commentaires et suggestions sur une version préliminaire de ce texte. 
L'éveil des historiens au monde de la santé s'inscrivait dans un bouillonnement d'idées neuves véhiculées par des chercheurs d'horizons disciplinaires variés et auxquelles adhéraient plusieurs organismes communautaires et groupes de gauche. Toute une série d'ouvrages consacrés à la santé et aux services de santé ont ainsi été publiés au Québec à la fin des années 1970 et dans la première moitié des années $1980^{2}$. Ils avaient en commun de remettre en cause la vision triomphaliste de la médecine qui avait prédominé jusqu'alors. Les chercheures féministes, par exemple, ont dénoncé une médicalisation à outrance de la maternité et la subordination des femmes dans l'univers sanitaire du travail. Certains se sont inquiétés de la déshumanisation des soins, de la hausse vertigineuse des coûts des équipements et des services, de la constitution d'un vaste appareil de contrôle social où la médecine jouait un rôle majeur. On s'interrogeait aussi sur l'ascension sociale de ceux qui avaient présidé à l'instauration de l'État-providence ou en avaient le plus bénéficié. Le système de santé québécois et ses réformes récentes se trouvaient de la sorte soumis à un regard éminemment critique. Ces préoccupations invitaient aussi, comme dans le reste du Canada et des sociétés occidentales, à creuser le passé pour y déterrer les racines des situations observées ou dénoncées, et allaient nourrir la réflexion historique.

Chez les historiens, l'effervescence caractéristique de pareille période de défrichements et l'urgence ressentie d'édifier un nouveau corps de connaissances étaient peu propices aux temps d'arrêt et aux vues d'ensemble. Le moment paraît venu de faire le point, de retracer l'évolution de la recherche en histoire de la santé au Québec ${ }^{3}$ en la situant dans les grands courants

2. Voir Barbara Ehrenreich et Deirdre English, Sorcières, sages-femmes et infirmières. Une histoire des femmes et de la médecine (Montréal, Les Éditions du Remue-ménage, 1976); Marc Renaud, The Political Economy of the Quebec State Interventions in Health : Reform or Revolution?, thèse de doctorat, University of Wisconsin, 1976; Frédéric Lesemann, Du pain et des services. La réforme de la santé et des services sociaux au Québec (Laval, Éditions coopératives Albert SaintMartin, 1981); Luciano Bozzini, Marc Renaud, Dominique Gaucher et Jaime Llambias-Wolff, Médecine et société. Les années 80 (Montréal, Éditions Saint-Martin, 1981); Barbara Ehrenreich et Deirdre English, Des experts et des femmes, 150 ans de conseils prodigués aux femmes (Montréal, Les Éditions du Remue-ménage, 1982); Jacques Dufresne, Fernand Dumont et Yves Martin, dir., Traité d'anthropologie médicale. L'institution de la santé et de la maladie (Sillery, Presses de l'Université du Québec, Institut québécois de recherche sur la culture, Presses universitaires de Lyon, 1985).

3. La publication, durant la décennie en cours, de textes à caractère historiographique et d'un recueil d'articles publiés antérieurement par divers auteurs, montre bien que ce besoin de faire le point est ressenti par plusieurs. Voir notamment Jacques Bernier, «La place de l'histoire de la médecine », Santé et société canadienne/Health and Canadian Society (SSC/HCS), 1,1 (1993) : 19-49; Peter Keating et Othmar Keel, dir., Santé et société au Québec : XIXee et XXe siècles (Montréal, Boréal, 1995); la première partie aussi de la chronologie de Denis Goulet et André Paradis, Trois siècles d'histoire médicale au Québec. Chronologie des institutions et des pratiques (1639-1939) (Montréal, vlb éditeur, 1992). Voir aussi, durant la décennie précédente : J. Bernier, «L'histoire de la médecine et de la santé au Canada français, 1976-1986 : aperçu historiographique », Scientia Canadensis, 11,1 (1987) : 28-33. 
historiographiques et d'en recenser les principaux acquis ${ }^{4}$. L'exercice devrait permettre d'identifier certaines lacunes ou fausses pistes et de relancer la recherche sur des voies nouvelles ou peu fréquentées.

Nous avons choisi de centrer notre réflexion sur l'histoire de la santé dans la ville. Les travaux qui ont marqué l'ouverture du champ durant les années 1970 s'inscrivaient dans celui des études urbaines, en même temps que dans celui de l'histoire des travailleurs ${ }^{5}$. Deux décennies plus tard, deux thèses de doctorat étaient soutenues qui abordaient la santé publique, respectivement dans la principale agglomération du Québec et dans deux petites villes ${ }^{6}$. Entre temps, bon nombre de travaux ont retenu la ville comme terrain d'enquête. Leurs auteurs se sont penchés sur toute une gamme d'objets d'étude que nous présenterons regroupés comme suit : les inégalités sociales et les rapports entre acteurs sociaux, la mise en place et l'organisation des services sanitaires, l'évolution de l'état de santé des populations et le rôle central des pôles urbains.

En première partie, une brève revue de l'évolution générale de l'histoire de la santé au Québec aidera à mieux saisir dans quelles perspectives chacun de ces objets d'étude a été abordé. Comment, du point de vue de la santé, les historiens ont-ils intégré à leurs problématiques la ville et sa population, ses institutions et ses services? De façon plus générale, quelle a été leur contribution à la compréhension de la société québécoise?

\section{L'ASCENDANT DE L'HISTOIRE SOCIALE ET LES ENJEUX DE LA RECHERCHE}

La santé a émergé comme objet d'étude chez les historiens québécois durant la décennie 1970. Leurs travaux, marqués par les grands courants

4. Deux ouvrages de synthèse récents présentent déjà à un public élargi les connaissances issues des deux dernières décennies de recherche : François Guérard, Histoire de la santé au Québec (Montréal, Boréal, 1996); Normand Séguin, dir., Atlas historique du Québec. L'institution médicale (Québec, Les Presses de l'Université Laval, 1998). Pour un panorama de l'histoire des sciences au Québec, voir Luc Chartrand, Raymond Duchesne et Yves Gingras, Histoire des sciences au Québec (Montréal, Boréal, 1987).

5. Ce sont les travaux de Terry Copp, Classe ouvrière et pauvreté, les conditions de vie des travailleurs montréalais, 1897-1929 (Montréal, Boréal Express, 1978), traduction d'un livre d'abord paru en anglais en 1974; et Martin Tétreault, L'état de santé des Montréalais, 1880-1914 (Montréal, Regroupement des chercheurs-chercheures en histoire des travailleurs et travailleuses du Québec, coll. «Études et documents », 1991).

6. Il s'agit des thèses de Benoît Gaumer, Le service de santé de la ville de Montréal. De la mise sur pied au démantèlement : 1865-1975, thèse de doctorat (sciences humaines appliquées), Université de Montréal, 1996; et de F. Guérard, La santé publique dans deux villes du Québec de 1887 à 1939. Trois-Rivières et Shawinigan, thèse de doctorat (histoire), Université du Québec à Montréal, 1993. 
historiographiques de l'heure, portent le sceau du renouvellement international de l'histoire de la santé inspiré par l'histoire sociale.

Un peu partout, l'histoire de la santé et de la médecine avait été pratiquée auparavant surtout par des médecins férus du passé de leur profession. Ils s'étaient penchés prioritairement sur les figures marquantes de l'avancement des connaissances médicales, sur des épidémies dévastatrices et sur diverses institutions. Quoique certains aient adopté une démarche plus critique, la plupart avaient choisi comme toile de fond à leurs travaux les progrès sanitaires de l'humanité à travers les siècles, progrès dont ils rendaient la médecine responsable. Le Québec n'échappe pas à la règle et possède également ses travaux précurseurs de médecinshistoriens ${ }^{7}$. Plusieurs membres du clergé aussi, tout autant désireux de célébrer l'œuvre de leurs prédécesseurs, ont publié des historiques d'hôpitaux et des communautés religieuses qui les dirigeaient ${ }^{8}$. Rédigés dans une perspective descriptive, tous ces ouvrages sont riches de matériaux que les historiens ont exploités par la suite.

Avec le déploiement de l'histoire sociale dans les années 1970, la santé est apparue rapidement comme un champ de recherche prometteur ${ }^{9}$. L'analyse des interactions entre les divers groupes sociaux y ouvre sur la société dans son ensemble, sur l'économie, sur le culturel et sur le politique. Pour les partisans de l'histoire sociale, attirés par une histoire dite totale ou du moins à caractère synthétique, il y avait là des perspectives alléchantes. Le champ de la santé se présentait à eux comme une voie parmi d'autres pour ouvrir une fenêtre sur des groupes précédemment ignorés des historiens : les «masses populaires », les femmes, les travailleurs, les démunis... On le voit, leur optique était tout autre que la mise en valeur de la médecine et de ses progrès. Ils allaient insister, notamment, sur le rôle déterminant joué par d'autres facteurs dans l'amélioration de l'état de santé des

7. On pense ici en particulier aux Michael Joseph et George Ahern, John Joseph Heagerty, Maude E. Abbott et Hugh Ernest MacDermot. Sur l'histoire de la médecine au Québec avant 1970, consulter J. Bernier, «La place de l'histoire de la médecine », loc. cit.

8. Les monographies d'hôpitaux et de communautés religieuses sont trop nombreuses pour être mentionnées ici.

9. Plusieurs textes traitent du renouveau de l'histoire de la médecine et de la santé. Voir notamment Ronald L. Numbers, «The History of American Medicine : A Field in Ferment », Reviews in American History, 10 (1982) : 245-263; Judith Walzer Leavitt, «Medicine in Context : A Review Essay of the History of Medicine », American Historical Review, 95 (déc. 1990) : 1471-1484; Olivier Faure, « Des médecins aux malades, tendances récentes en histoire sociale de la santé », dans Michel Lagrée et François Lebrun, dir., Pour l'histoire de la médecine. Autour de l'œuvre de Jacques Léonard (Rennes, Presses universitaires de Rennes, 1994), 59-69; Wendy Mitchinson, « Medical Historiography in English Canada », SSC/HCS, 1,1 (1993) : 205-227. 
populations ${ }^{10}$ et mettre en scène une profession médicale soucieuse d'acquérir une situation sociale privilégiée et de régir l'ensemble du champ sanitaire ${ }^{11}$. S'attaquer aux inégalités sociales devant la maladie et la mort, scruter sans complaisance la montée de groupes professionnels comme celui des médecins ou l'évolution des rôles respectifs de l'Église et de l'État dans le cas du Québec, dénoncer la place et le sort réservés aux femmes dans le monde de la santé, autant d'éléments de programme qui allaient creuser le lit d'un renouveau historiographique.

D'entrée de jeu, et assez massivement par la suite, les historiens de la santé au Québec empruntent la voie de l'histoire sociale ${ }^{12}$ ou, à tout le moins, s'en réclament. Durant la décennie 1980, le rythme des publications s'accélère et la thématique s'élargit considérablement, alors que l'histoire de la santé et de la médecine se constitue en champ autonome : elle possède désormais ses propres historiens spécialistes qui lui consacrent l'essentiel de leurs recherches, l'intègrent à leur enseignement et y orientent plusieurs étudiants de maîtrise et de doctorat. Bon nombre aborderont la santé dans la ville ou se pencheront sur des acteurs sociaux et des services sanitaires urbains, ce qui s'explique assez aisément. C'est d'abord dans les villes que les réformes sanitaires ont été mises en œuvre. La grande majorité des services, des médecins et des travailleurs de la santé en général s'y trouvent concentrés. De plus, fort peu de sources livrent des informations sur la santé en milieu rural. Par ailleurs, le développement rapide des études urbaines durant la même période appelait à situer une large variété d'objets d'étude dans le cadre de la ville ${ }^{13}$.

10. Les thèses de Thomas McKeown sur les causes de l'amélioration de l'état de santé des populations ont été abondamment citées : Thomas McKeown, The Modern Rise of Population (London, Edward Arnold, 1976); Thomas McKeown, The Role of Medicine, Dream, Mirage, or Nemesis? (Princeton, Princeton University Press, 1979).

11. À titre d'exemples d'une vaste littérature, mentionnons : Irving Kenneth Zola, « Medicine as an Institution of Social Control », dans John Ehrenreich, The Cultural Crisis of Modern Medicine (New York, Monthly Review Press, 1978), 80-100; Jacques Léonard, La médecine entre les pouvoirs et les savoirs, histoire intellectuelle et politique de la médecine française au XIXe siècle (Paris, Aubier, Collection historique, 1981); Paul Starr, The Social Transformation of American Medicine. The Rise of a Sovereign Profession and the Making of a Vast Industry (New York, Basic Books, 1982).

12. André Paradis, par exemple, l'un des pionniers de l'histoire de la santé au Québec, après avoir tâté d'autres approches au cours des années 1970, en est venu à promouvoir l'histoire sociale : «L'histoire de la médecine : une porte ouverte sur l'histoire sociale », Revue d'histoire de l'Amérique française (RHAF), 42,1 (été 1988) : 73-83. Fait significatif, lorsque deux historiens ont publié en 1995 un recueil des textes qu'ils jugeaient les plus représentatifs des nouvelles problématiques en histoire de la médecine et de la santé au Québec, ils l'ont coiffé d'un titre qui commence par « Santé et Société ». Voir P. Keating et O. Keel, dir., op. cit.

13. Sur le développement de l'histoire urbaine au Canada et au Québec à cette époque, consulter Paul-André Linteau et Alan F. J. Artibise, L'évolution de l'urbanisation au Canada : une analyse des perspectives et des interprétations (Winnipeg, The Institute of Urban Studies, University of 
La décennie actuelle, enfin, s'avère particulièrement féconde : les publications abondent ${ }^{14}$. La poursuite de nombreuses recherches et l'ouverture de nouveaux chantiers suggèrent pour les années prochaines une progression encore rapide des connaissances. La remarquable vitalité du champ de recherche vient d'être consacrée par la tenue, sous le thème "Médecine, santé et sociétés », du congrès annuel de l'Institut d'histoire de l'Amérique française. Le programme du congrès a témoigné d'un certain engouement pour la santé : près de quarante présentations s'inscrivaient dans le thème retenu. La présence non seulement d'historiens, mais aussi de plusieurs chercheurs d'autres disciplines - archéologie, sociologie, archivistique par exemple - augure bien pour les développements à venir. Il paraît en effet souhaitable d'encourager les collaborations interdisciplinaires si l'on veut que l'histoire de la santé élargisse encore ses horizons et affine ses méthodes.

\section{LA CONCENTRATION DES TRAVAUX SUR LA MÉTROPOLE ET SUR LES XIX ${ }^{e}$ ET XX ${ }^{e}$ SIÈCLES}

Dans l'ensemble, pour ce qui a trait à l'histoire de la santé dans la ville, la période qui va du milieu du XIX ${ }^{\mathrm{e}}$ siècle à 1945 s'avère, de loin, la mieux couverte. Durant cette période, le processus d'urbanisation et d'industrialisation s'enclenche et, graduellement, transforme en profondeur la société québécoise. Le laisser-aller initial dans le domaine sanitaire laisse alors place à l'organisation, par étapes, de tout un ensemble de services qui visent l'amélioration de la santé des citadins, d'abord par l'assainissement du milieu urbain, puis par des programmes de médecine préventive de plus en plus ambitieux. De surcroît, la mortalité subit durant la première moitié $\mathrm{du} \mathrm{XX}^{\mathrm{e}}$ siècle un recul d'une ampleur sans précédent. Il était normal et souhaitable qu'une telle période de chambardements reçoive une attention particulière. Quant à l'enrichissement de nos connaissances sur la santé dans la ville pré-industrielle, il provient généralement soit de chercheurs qui ont inclu des considérations d'ordre sani-

\footnotetext{
Winnipeg, rapport $\left.n^{\circ} 5,1984\right)$. À l'échelle internationale, diverses études portant sur l'hygiène publique en milieu urbain ou sur le monde hospitalier allaient inspirer les chercheurs québécois. Voir, par exemple, aux États-Unis : Judith Walzer Leavitt, The Healthiest City. Milwaukee and the Politics of Health Reform (Princeton, Princeton University Press, 1982); Morris J. Vogel, The Invention of the Modern Hospital : Boston, 1870-1930 (Chicago, University of Chicago Press, 1980); David Rosner, A Once Charitable Enterprise. Hospitals and Health Care in Brooklyn and New York, 18851915 (Cambridge, Cambridge University Press, 1982); Charles E. Rosenberg, The Care of Strangers. The Rise of America's Hospital System (New York, Basic Books, 1987); Rosemary Stevens, In Sickness and in Wealth. American Hospitals in the Twentieth Century (New York, Basic Books, 1989).

14. L'histoire de la santé a été identifiée comme l'un des champs de recherche qui ont connu le développement le plus rapide de 1992 à 1996. Voir Gérard Bouchard, «L'histoire sociale au Québec. Réflexion sur quelques paradoxes », RHAF, 51,2 (automne 1997) : 243-269.
} 
taire dans des travaux portant sur la ville ${ }^{15}$, soit d'historiens de la santé qui n'avaient pas le monde urbain comme point de mire ${ }^{16}$.

Il n'y a rien d'étonnant non plus à ce qu'une majorité de travaux ait élu l'île de Montréal comme terrain d'enquête. Non seulement représente-t-elle, déjà au milieu du XIX ${ }^{\mathrm{e}}$ siècle, la principale concentration de population, mais en outre, elle en vient à rassembler plus que sa part de services. De plus, on y trouve aujourd'hui plusieurs historiens de la santé qui peuvent être tentés, de même que leurs étudiants, d'exploiter des fonds d'archives situés à proximité. Résultat de cette abondante moisson montréalaise, lorsqu'un spécialiste de l'histoire urbaine comme PaulAndré Linteau publie une monographie sur l'histoire de la métropole, il est en mesure de consacrer plusieurs pages à la santé17.

À l'instar des terrains et des périodes d'enquête, certaines avenues de recherche ont été privilégiées, alors que d'autres sont demeurées plus ou moins en friche. Voici, sommairement tracé, un état des principaux acquis et un aperçu de certaines lacunes.

\section{LES INÉGALITÉS ET LES RAPPORTS ENTRE ACTEURS SOCIAUX}

Comme l'histoire de la santé dans la ville s'est pour une bonne part développée de concert avec le courant de l'histoire sociale, les rapports entre groupes sociaux sont devenus une dimension essentielle dans plusieurs travaux. La promotion et la confrontation des intérêts des différents acteurs se substituaient aux progrès de la médecine comme principal moteur du changement.

Dans un premier temps, les praticiens de l'histoire des travailleurs ont vu dans les rapports entre les classes sociales le principe générateur des écarts observés sur le plan sanitaire aux $\mathrm{XIX}^{\mathrm{e}}$ et $\mathrm{XX}^{\mathrm{e}}$ siècles. L'examen de l'état de santé des populations allait jeter un éclairage cru sur les iné-

15. Voir, par exemple, John Hare, Marc Lafrance et David-Thiery Ruddel, Histoire de la ville de Québec, 1608-1871 (Montréal/Hull, Boréal/Musée canadien des civilisations, 1987). Sur l'hygiène dans la ville en Nouvelle-France, voir André Lachance, La vie urbaine en Nouvelle-France (Montréal, Boréal Express, coll. «Histoire et sociétés », 1987); Louise Dion, L'épidémie de variole de l'hiver 1702-1703 dans la ville de Québec, mémoire de maîtrise (histoire), Université de Sherbrooke, 1981.

16. Parmi les travaux concernés ici, mentionnons, Rénald Lessard, Se soigner au Canada aux XVII ${ }^{e}$ et XVIII ${ }^{e}$ siècles (Hull, Musée canadien des civilisations, coll. « Mercure », 1989); id., Pratique et praticiens en contexte colonial : le corps médical canadien aux $17^{e}$ et $18^{e}$ siècles, thèse de doctorat (histoire), Université Laval, 1994; id., «Les soins de santé au Canada aux XVII et XVIII siècles », dans N. Séguin, dir., op. cit., 3-35; Toby Gelfand, « Who Practised Medicine in New France? A Collective Portrait », dans Charles-G. Roland, dir., Health, Disease and Medicine : Essays in Canadian History (Toronto, The Hannah Institute for the History of Medicine, 1984), 16-35; id., « Medicine in New France », dans Ronald L. Numbers, dir., Medicine in the New World: New Spain, New France, and New England (Knoxville, University of Tennessee Press, 1987), 64-100.

17. Paul-André Linteau, Histoire de Montréal depuis la Confédération (Montréal, Boréal, 1992). 
quités sociales dans la ville industrielle. Pour Montréal, Terry Copp et Martin Tétreault ${ }^{18}$ ont montré que, durant les dernières décennies du $\mathrm{XIX}^{\mathrm{e}}$ siècle et les premières du $\mathrm{XX}^{\mathrm{e}}$, les écarts socio-économiques entre les groupes bien nantis et les travailleurs s'y exprimaient dans une mortalité nettement différenciée ${ }^{19}$. Les premiers, ont-ils souligné, habitaient dans des quartiers convenablement équipés sur le plan sanitaire, alors que les seconds s'entassaient dans des secteurs mal desservis et insalubres. Ces historiens ont constaté également que la mortalité était nettement plus sévère chez les francophones que chez les anglophones et ils ont attribué cela à une surreprésentation des francophones dans le groupe des travailleurs, soumis à des conditions de vie désavantageuses.

D'autres chercheurs ont par la suite souligné que les différences socio-économiques ne recoupaient qu'approximativement les écarts sanitaires observés et qu'il fallait par conséquent recourir à d'autres clivages. Jean-Claude Robert ${ }^{20}$, Sherry Olson et Patricia Thornton ${ }^{21}$ notamment, ont suggéré que les différences culturelles entre les groupes linguistiques et ethniques, leurs modes de vie respectifs, leurs habitudes alimentaires pouvaient expliquer certaines particularités de la mortalité montréalaise au XIX ${ }^{\mathrm{e}}$ siècle. Ainsi, il semble que les écarts de mortalité infantile entre francophones catholiques, Irlandais de souche et angloprotestants soient attribuables à des habitudes distinctes quant à la durée de l'allaitement maternel.

Les clivages ethniques, linguistiques et religieux, faut-il préciser, se sont imposés dans nombre d'études, aussi bien en ce qui a trait à l'organisation des services qu'à l'état de santé. Invariablement, dès

18. T. Copp, op. cit.; id., « The Health of the People : Montreal in the Depression Years », dans D. A. E. Shephard et Andrée Lévesque, dir., Normand Bethune, his Times and his Legacy (Ottawa, Canadian Public Health Association, 1982), 129-137; Martin Tétreault, « Les maladies de la misère. Aspects de la santé publique à Montréal 1880-1914 », RHAF, 36,4 (mars 1983) : 507-526; id., «L'état de santé... », op. cit. Voir aussi Louise Dechêne et Jean-Claude Robert, « Le choléra de 1832 dans le Bas-Canada : mesure des inégalités devant la mort », dans Hubert Charbonneau et André LaRose, dir., The Great Mortalities : Methodological Studies of Demographic Crises in the Past (Liège, Ordina, 1979), 229-255.

19. Ils reprenaient un constat exprimé par nombre de médecins qui, depuis le XIX ${ }^{\mathrm{e}}$ siècle, s'étaient tournés vers l'épidémiologie et la statistique démographique : celui de l'inégalité sociale devant la mort.

20. Jean-Claude Robert, «The City of Wealth and Death : Urban Mortality in Montreal, 1821-1871 », dans Wendy Mitchinson et Janice Dickin McGinnis, dir., Essays in the History of Canadian Medicine (Toronto, McClelland \& Stewart, 1988), 18-38.

21. Patricia A. Thornton, Sherry Olson et Q. Thuy Thach, « Dimensions sociales de la mortalité infantile à Montréal au milieu du XIX ${ }^{\mathrm{e}}$ siècle », Annales de démographie historique (1988) : 299-325; P. A. Thornton et S. Olson, « Family Contexts of Fertility and Infant Survival in Nineteenth-Century Montreal », Journal of Family History, 16,4 (1991) : 401-417; S. Olson et P. Thornton, «Familles montréalaises du XIX ${ }^{\mathrm{e}}$ siècle : trois cultures, trois trajectoires », Cahiers québécois de démographie, 21,2 (automne 1992) : 51-75. 
lors que Montréal constitue le territoire d'enquête, ces clivages ressortent : les anglo-protestants y ont leur faculté de médecine de réputation internationale, ils façonnent un dispositif hospitalier complet parallèle à celui des franco-catholiques ${ }^{22}$ et ils se donnent leurs propres services sociaux, dispensaires et cliniques de « gouttes de lait». À Sherbrooke et à Québec, ils mettent sur pied un hôpital général distinct, sans compter que, dans plusieurs petites villes, c'est la direction anglophone de grandes entreprises qui établit le seul hôpital dont dispose la population. La communauté juive de la métropole, bien organisée, ouvre un hôpital général, de même qu'un sanatorium antituberculeux. C'est elle qui présente les taux de mortalité les plus bas. En période de crise, comme lors de l'épidémie de variole de 1885, il arrive que les tensions entre anglophones et francophones deviennent singulièrement vives.

Suivant une ligne de partage tout autre, un axe de recherche s'est imposé : celui de l'expérience spécifique des femmes vis-à-vis de la santé $^{23}$. Leur activité dans les services de santé a fait l'objet de nombre d'études. On a d'abord mis l'accent sur les démarches des infirmières pour obtenir une reconnaissance professionnelle et sur leur sujétion aux médecins, puis sur le rôle majeur que les femmes ont joué dans l'implantation de nouveaux programmes d'hygiène publique, de même que dans les services hospitaliers et sociaux ${ }^{24}$. La marginalisation des

22. F. Guérard, « Les grands appareils sanitaires », dans N. Séguin, dir., op. cit., 75-115.

23. Denyse Baillargeon développe, dans un autre article de ce numéro de la revue, la question de l'apport de l'histoire des femmes dans le champ de la santé au Québec. J'y renvoie le lecteur avec plaisir et ne relève ici que certains éléments qui concernent directement la ville et la santé.

24. Yolande Cohen et Michèle Dagenais, « Le métier d'infirmière : savoirs féminins et reconnaissance professionnelle », RHAF, 41,2 (automne 1987) : 155-177; Y. Cohen et Michèle Gélinas, «Les infirmières hygiénistes de la ville de Montréal : du service privé au service civique », Histoire sociale/Social History (HS/SH), 22,44 (novembre 1989) : 219-246; Y. Cohen et Louise Bienvenue, «Émergence de l'identité professionnelle chez les infirmières québécoises, 1890-1927 », Canadian Bulletin of Medical History/Bulletin canadien d'histoire de la médecine (CBMH/BCHM), 11 (1994) : 119-151; Y. Cohen et Éric Vaillancourt, «L'identité professionnelle des infirmières canadiennes-françaises à travers leurs revues », RHAF, 50,4 (printemps 1997) : 537-570; Johanne Daigle, L'émergence et l'évolution de l'alliance des infirmières de Montréal : 1946-1966, mémoire de maîtrise (histoire), Université du Québec à Montréal, 1983; id., «La syndicalisation des infirmières », dans Marie Lavigne et al., dir., Travailleuses et féministes, les femmes dans la société québécoise (Montréal, Boréal Express, 1983), 115-138; id., Devenir infirmière : le système d'apprentissage et la formation professionnelle à l'Hôtel-Dieu, 1920-1970, thèse de doctorat (histoire), Université du Québec à Montréal, 1990; J. Daigle, Nicole Rousseau et Francine Saillant, « Des traces sur la neige... La contribution des infirmières au développement des régions isolées du Québec au XX ${ }^{\mathrm{e}}$ siècle », Recherches féministes $(R F)$ 6,1 (1993) : 93-103; J. Daigle et N. Rousseau, « Le service médical aux colons. Gestation et implantation d'un service infirmier au Québec (1932-1943) », RHAF, 52,1 (été 1998) : 47-72; André Petitat, Les infirmières. De la vocation à la profession (Montréal, Boréal, 1989); Éric Vaillancourt, Les rapports médecins-infirmières au Canada et au Québec : analyse de l'interdiscursif à travers leurs revues professionnelles (1867-1920), mémoire de maîtrise (histoire), Université du Québec à Montréal, 1995. 
sages-femmes par les médecins et la médicalisation de la maternité25, le travail des religieuses ${ }^{26}$ et des bénévoles ${ }^{27}$, certaines occupations paramédicales ${ }^{28}$ ont également retenu l'attention. Dans la perspective des études spécifiquement urbaines, mentionnons les travaux sur les interventions de santé publique qui visaient à inculquer aux mères de nouvelles notions d'hygiène infantile. Denyse Baillargeon ${ }^{29}$ a mis en lumière le rôle de promoteur joué par certains groupes de femmes dans l'ouverture des premières cliniques de « gouttes de lait » à Montréal, puis leur éviction comme organisatrices. Elle a aussi montré que la fréquentation

25. Marie-Josée Blais, Le transfert hospitalier de l'accouchement au Québec, 1930-1960, mémoire de maîtrise (histoire), Université de Montréal, 1995; Louise Brodeur, Les débuts de la spécialisation de la gynécologie au Québec, 1880-1920. Médicalisation de la femme et consolidation de la profession médicale, mémoire de maitrise (histoire), Université de Montréal, 1991; Danielle Gauvreau, «Donner la vie et en mourir : la mortalité des femmes en couches au Québec avant 1960 », dans Denis D. Cordell et al., dir., Population, reproduction, sociétés : perspectives et enjeux de démographie sociale : mélanges en l'honneur de Joel W. Gregory (Montréal, Presses de l'Université de Montréal, 1993); Hélène Laforce, Histoire de la sage-femme dans la région de Québec (Québec, Institut québécois de recherche sur la culture, 1985); Michel O'Neill, Accoucher autrement : repères historiques sociaux et culturels de la grossesse et de l'accouchement (Montréal, Éditions Albert Saint-Martin, 1987).

26. Voir Y. Cohen, « La contribution des Sœurs de la Charité à la modernisation de l'Hôpital Notre-Dame de Montréal», Canadian Historical Review (CHR) 77,2 (juin 1996) : 185-220; Danielle Juteau et Nicole Laurin-Frenette, Un métier et une vocation : le travail des religieuses au Québec de 1901 à 1971 (Montréal, Presses de l’Université de Montréal, coll. «Trajectoires sociales », 1997); Nicole Laurin, D. Juteau et Lorraine Duchesne, À la recherche d'un monde oublié. Les communautés religieuses de femmes au Québec de 1900 à 1970 (Montréal, Le Jour, 1991).

27. Voir Aline Charles, Travail d'ombre et de lumière : le bénévolat féminin à l'Hôpital Sainte-Justine, 1907-1960 (Québec, Institut québécois de recherche sur la culture, coll. « Edmond-deNevers ", n 9, 1990); id., Travail et vieillesses féminines dans les hôpitaux québécois : 1940-1980, thèse de doctorat (histoire), Université du Québec à Montréal, 1997; Nathalie Côté, Le rôle central des femmes bénévoles au sein de l'Assistance maternelle et de la Goutte de lait de Sherbrooke (19221960), mémoire de maîtrise (histoire), Université de Sherbrooke, 1993.

28. Nadia Fahmy-Eid et A.Charles, « Savoir contrôlé ou pouvoir confisqué? La formation des filles en technologie médicale, réhabilitation et diététique, 1940-1970 », $R F, 1,1$ (1988) : 5-30; id., « Raison d'État ou du plus fort? La diététique et la physiothérapie en quête d'une pratique exclusive au Québec, 1950-1980», HS/SH, 26,51 (mai 1993) : 95-113; N. Fahmy-Eid et al., Femmes, santé et professions : histoire des diététistes et des physiothérapeutes au Québec et en Ontario, 1930-1980 (Montréal, Fides, 1997). Sur les employées de soutien des hôpitaux, voir Michèle Gélinas, Les travailleuses de soutien des hôpitaux du Québec, mémoire de maîtrise (histoire), Université de Montréal, 1985.

29. D. Baillargeon, « Care of Mothers and Infants in Montreal Between the Wars : The Visiting Nurses of the Metropolitan Life, Milk Deposits and assistance maternelle », dans Diane Dodd et Deborah Gorham dir., Caring and Curing : Historical Perspectives on Women and Healing in Canada (Ottawa, Presses de l'Université d'Ottawa, 1994), 163-182; id., «Fréquenter les "Gouttes de lait". L'expérience des mères montréalaises, 1910-1965 », RHAF, 50,1 (été 1996) : 29-68; id. «Les rapports médecins-infirmières et l'implication de la Métropolitaine dans la lutte contre la mortalité infantile, 1909-53 », CHR, 77,1 (mars 1996) : 53-59; id., « Gouttes de lait et soif de pouvoir : les dessous de la lutte contre la mortalité infantile à Montréal, 1910-1953 », CBMH/BCHM, 15,1 (1998). Sur les groupes de femmes actifs dans la promotion de l'hygiène publique, voir aussi Louise Bienvenue, Le rôle du Victorian Order of Nurses dans la croisade hygiéniste montréalaise (18971925), mémoire de maîtrise (histoire), Université du Québec à Montréal, 1995. 
de ces cliniques, ou leur non-fréquentation, étaient dans bien des cas motivées par des facteurs qui relevaient tout autant de la socialisation de la femme au foyer que de la santé. Point à souligner, l'histoire des femmes est peut-être la mieux parvenue à amorcer une réflexion sur l'usage fait par la population des services de santé.

$\mathrm{Au}$ fur et à mesure que les historiens fouillaient plus en profondeur leurs terrains d'enquête, ils ont ressenti le besoin de mieux camper le rôle, les intérêts et les prises de position de groupes assez restreints, en particulier les médecins et les infirmières, ou encore le clergé. On a ainsi fait ressortir les stratégies que chacun déployait pour occuper l'espace sanitaire urbain, le dominer ou simplement y faire sa place. À cet égard, bien qu'elles ne soient souvent pas centrées sur le monde urbain, les études de Yolande Cohen et de Johanne Daigle sur les infirmières ${ }^{30}$, de Johanne Colin sur les pharmaciens ${ }^{31}$, de Jacques Bernier $^{32}$, de Denis Goulet ${ }^{33}$ et de Claudine Pierre-Deschênes ${ }^{34}$ sur la profession médicale, de plusieurs auteurs sur l'enseignement de la

30. Voir la note 24 .

31. Johanne Collin, Évolution de la profession pharmaceutique au Québec au $X X^{e}$ siècle : une analyse du rapport entre les transformations de la pratique et la féminisation du corps professionnel, thèse de doctorat (histoire), Université du Québec à Montréal, 1991; id., «Les femmes dans la profession pharmaceutique au Québec : rupture ou continuité », $R F, 5,2$ (1992) : 31-56; J. Collin et Denis Béliveau, Histoire de la pharmacie au Québec (Montréal, Musée de la pharmacie au Québec, SABEX et Rougier inc., 1994); id., Changement d'ordonnance. Mutations professionnelles, identité sociale et féminisation de la profession pharmaceutique au Québec, 1940-1980 (Montréal, Boréal, 1995); id., "Genèse d'une profession : les pharmaciens au Québec au XIX ${ }^{\mathrm{e}}$ siècle », CBMH/BCHM, 14,2 (1997) : 241-262. Voir aussi Yves Sévigny, L'image du pharmacien et les pratiques pharmaceutiques dans la presse de Sherbrooke (1837-1908), mémoire de maîtrise (histoire), Université de Sherbrooke, 1994.

32. J. Bernier, «Les praticiens de la santé au Québec, 1871-1921 : quelques données statistiques », Recherches sociographiques $(R S), 20,1$ (janvier-avril 1979) : 41-58; id., « François Blanchet et le mouvement réformiste en médecine au début du XIX ${ }^{\mathrm{e}}$ siècle », RHAF, 34,2 (septembre 1980) : 223-244; id., « Le corps médical québécois à la fin du XVIII ${ }^{\mathrm{e}}$ siècle », dans Charles-G. Roland, dir., Health, Disease and Medicine : Essays in Canadian History (Toronto, The Hannah Institute for the History of Medicine, 1984), 36-64; id., «L'intégration du corps médical québécois à la fin du XIX ${ }^{\mathrm{e}}$ siècle », Historical Reflections, 10 (1983) : 91-113; id., «Vers un nouvel ordre médical : les origines de la corporation des médecins et chirurgiens du Québec », RS, 22,2 (1981) : 307 330; id., La médecine au Québec. Naissance et évolution d'une profession (Québec, Les Presses de l'Université Laval, 1988).

33. Voir, parmi d'autres titres également mentionnés dans l'article, D. Goulet, Histoire du Collège des médecins du Québec, 1847-1997 (Montréal, Collège des médecins du Québec, 1997); $i d$., «La structuration de la pratique médicale, 1800-1940», dans N. Séguin, op. cit.

34. Claudine Pierre-Deschênes, La tuberculose au Québec au début du XX $X^{e}$ siècle : problème social et réponse réformiste, mémoire de maîtrise (histoire), Université du Québec à Montréal, 1980; id., « Santé publique et organisation de la profession médicale au Québec, 1870-1918 », RHAF, 35,3 (décembre 1981) : 355-375. 
médecine ${ }^{35}$ ou sur les thérapeutes concurrents ${ }^{36}$ constituent d'excellents exemples.

Mais ces groupes même se sont trouvés fragmentés : les médecins sont apparus divisés entre anglophones et francophones quant à la législation et à la formation professionnelle, entre élites des grands centres et praticiens des régions, entre partisans et adversaires de l'application de certaines mesures, entre omnipraticiens et spécialistes, etc.; on a constaté que les infirmières étaient partagées entre des associations aux objectifs parfois discordants, et que les infirmières religieuses et les laïques ne bénéficiaient pas du même statut dans l'hôpital; quant au clergé francophone, il avait aussi ses dissensions internes, dans la mesure où l'épiscopat, les communautés religieuses et les curés des paroisses ne partageaient pas toujours une même vision $^{37}$. Ce découpage en groupes et en sous-groupes toujours plus

35. Concernant l'enseignement de la médecine, mentionnons : J. Bernier, « La standardisation des études médicales et la consolidation de la profession dans la deuxième moitié du XIX ${ }^{\mathrm{e}}$ siècle», RHAF, 37,1 (décembre 1983) : 51-66; Georges Desrosiers, B. Gaumer et O. Keel, «L'évolution des structures de l'enseignement universitaire spécialisé de santé publique au Québec : 1899-1970 », CBMH/BCHM, 6,1 (été 1989) : 3-26; id., «L'introduction de l'enseignement de l'hygiène au Québec », dans Jean-Pierre Goubert et O. Keel, dir., Polysémie de la santé. Institutions et pratiques sociales en France et au Québec (1750-1980) (Montréal, EHESS/CNRS, Cahiers du Centre de recherches historiques, $\mathrm{n}^{\circ}$ 12, 1994), 85-104; G. Desrosiers et al., «Contribution de l'École d'hygiène de l'Université de Montréal à un enseignement francophone de santé publique 1946-1970», RHAF, 47,3 (hiver 1994) : 323-347; G. Desrosiers, Histoire du département de médecine sociale et préventive de la Faculté de médecine de l'Université de Montréal (Montréal, Département de médecine sociale et préventive, Université de Montréal, 1996); Céline Déziel, L'enseignement clinique à l'Hôpital Notre-Dame de 1880 à 1924, mémoire de maîtrise (histoire), Université de Montréal, 1992; Dominique Gaucher, « La formation des hygiénistes à l'Université de Montréal, 1910-1975 : de la santé publique à la médecine préventive », RS, 20 (1979) : 59-85; D. Goulet, Histoire de la faculté de médecine de l'Université de Montréal 1843-1993 (Montréal, vlb éditeur, coll. «Études québécoises », 1993); id., « L'enseignement médical à l'Université de Montréal : du modèle européen au modèle américain (1843-1980) », dans J.-P. Goubert et O. Keel, dir., op. cit., 117-130; id., « La structuration de la pratique médicale, 1800-1940», dans N. Séguin, dir., op. cit., 117-153; Joseph Hanaway et Richard Cruess, McGill Medicine, 1 : The First Half Century, 1829-1885 (Montréal, McGillQueen's University Press, 1996); George Weisz, «Origines géographiques et lieux de pratique des diplômés en médecine au Québec de 1834 à 1939 », dans Marcel Fournier, Y. Gingras et O. Keel, dir., Sciences et médecine au Québec, perspectives socio-historiques (Montréal, Institut québécois de recherche sur la culture, 1987), 129-170. Mentionnons aussi une référence plus ancienne : Charles-Marie Boissonnault, Histoire de la faculté de médecine de Laval (Québec, Les Presses de l'Université Laval, 1953).

36. Voir, par exemple, Jean-Pierre Robitaille, «L’homéopathie au Québec (1840-1904) : l'institutionnalisation d'une pratique médicale controversée », RHAF, 50,3 (hiver 1997) : 347-374.

37. Voir, par exemple, D. Juteau et N. Laurin, « La sécularisation et l'étatisation du secteur hospitalier au Québec de 1960 à 1966 », dans Robert Comeau, dir., Jean Lesage et l'éveil d'une nation. Les débuts de la Révolution tranquille (Sillery, Presses de l'Université du Québec, 1988), 155-167; F. Guérard, La santé publique..., op. cit.; François Rousseau, La croix et le scalpel. Histoire des Augustines et de l'Hôtel-Dieu de Québec, 2 : 1892-1989 (Sillery, Septentrion, 1994). 
fins n'est pas propre, loin s'en faut, à l'histoire de la santé au Québec : il s'est exprimé dans bien d'autres champs de recherche et d'autres provinces ou pays, et il a soulevé nombre de questions d'ordre épistémologique. Ici, cette fragmentation s'inscrit dans le vaste mouvement historiographique qui a remodelé le regard porté sur le Québec francophone d'avant 1960, auparavant perçu comme monolithique et refermé sur lui-même, particulièrement sur le plan des idéologies. À ce titre, elle apporte des arguments de poids à l'historiographie québécoise des dernières décennies — que Ronald Rudin a dénoncée comme «révisionniste ${ }^{38}$ »-, en révélant une diversité d'intérêts et d'opinions jusqu'alors méconnue, et en amenant à réévaluer le rapport des Québécois à la modernité, y compris pour les groupes apparemment les plus enclins à un certain conservatisme. On pense ici bien sûr au clergé ou à cette petite bourgeoisie dont même les plus traditionalistes militent parfois en faveur de réformes sanitaires progressistes.

De fait, l'étude des discours sur la santé et la ville a dévoilé un Québec ouvert sur le monde et qui participe aux courants d'idées internationaux. Tout un discours médicosocial, importé de l'Europe et des États-Unis et adapté au contexte québécois, promeut les intérêts de la profession médicale, tout en rejoignant les préoccupations des groupes dirigeants ${ }^{39}$. Ainsi, les hygiénistes et les médecins d'hôpitaux ciblent les milieux politiques et d'affaires en se faisant les défenseurs de la productivité, de la puissance et de la sécurité de la nation.

On a découvert que les partisans des politiques traditionalistes au Québec avaient été sensibles à cette argumentation. À les en croire, la ville était le tombeau de la « race », ce que semblaient confirmer les taux élevés de la mortalité urbaine, et il fallait endiguer l'exode rural. Mais leur volonté de promouvoir la catholicité francophone en Amérique allait les amener à appuyer un réaménagement sanitaire du milieu urbain en vue d'améliorer l'état de santé de la population. Le clergé et ses alliés ont, notamment, collaboré aux grandes campagnes d'hygiène publique lancées après la Première Guerre mondiale. Certes, ils l'ont fait bien souvent au nom d'un assainissement moral, par exemple lorsqu'il était question de tuberculose, de maladies vénérien-

38. Les idées les plus récentes de Ronald Rudin sur le « révisionnisme » peuvent être trouvées dans Ronald Rudin, Faire de l'histoire au Québec (Sillery, Septentrion, 1998).

39. C. Pierre-Deschênes, loc. cit.; Michèle Comeau, Discours et pouvoir de la profession médicale canadienne-française 1930-1950, mémoire de maîtrise (histoire), Université du Québec à Montréal, 1989; F. Guérard, «Les grands appareils sanitaires », dans N. Séguin, dir., op. cit., $75-115$. 
nes et, dans une autre perspective, de la santé des femmes ${ }^{40}$. De plus, ils se sont montrés réticents face à la prise en charge de certains services de santé par l'État et ont longtemps prôné la supériorité de l'initiative privée. Mais la défense même de ces positions allait les obliger à favoriser la modernisation des services offerts par les groupes philanthropiques et par les communautés religieuses, de sorte que l'intervention de l'État n'apparaisse pas nécessaire, inéluctable ${ }^{41}$.

Les historiens ont en somme entrepris un examen relativement systématique des rapports entre groupes sociaux actifs dans le monde de la santé. Au passage, nombre d'idées reçues ont été vigoureusement combattues. Certaines grandes questions qui hantent l'historiographie du Québec mériteraient toutefois d'être révisées à la lumière des découvertes des dernières décennies. Ainsi, le champ de la santé n'apparaît-il pas comme un lieu privilégié pour en arriver à une meilleure compréhension du rôle et de l'influence du clergé catholique dans la société québécoise? On mesure mieux aujourd'hui les limites de son pouvoir, dont les contours sont notamment dessinés par l'ascension sociale des médecins et par l'entrée en scène de l'État. Il n'en reste pas moins à définir des interprétations qui tiennent compte de ces limites, tout en expliquant le maintien prolongé d'une position de force.

\section{RÉFORMES ET ORGANISATION DES SERVICES}

Les nombreux travaux consacrés à l'organisation des services de santé ont eux aussi révélé la portée remarquable des influences européennes et américaines et ce, de la Nouvelle-France jusqu' au $\mathrm{XX}^{\mathrm{e}}$ siècle. Avant les dernières décennies du $\mathrm{XIX}^{\mathrm{e}}$ siècle, les interventions d'hygiène publique dans les villes étaient demeurées sporadiques. À l'exception des quarantai-

40. Sur la perception de la santé des femmes, voir, par exemple, Suzanne Commend, De la femme déchue à la femme infectieuse : perception sociale et répressions de la prostitution montréalaise pendant la Seconde Guerre mondiale, mémoire de maîtrise (histoire), Université de Montréal, 1996; Carole Dion, La femme et la santé de la famille au Québec, 1890-1914, mémoire de maîtrise (histoire), Université de Montréal, 1984; Andrée Lévesque, « Mères ou malades : les Québécoises de l'entre-deux-guerres vues par les médecins », RHAF, 38,1 (juin 1984) : 23-37; Doreen McCaughry, Le discours sur la femme dans la littérature médicale québécoise, 1880-1914, mémoire de maîtrise (histoire), Université d'Ottawa, 1983; Hélène Naubert, Maternité et pathologie : étude du discours médical sur la grossesse et l'accouchement au Québec (1870-1900), mémoire de maîtrise (études québécoises), Université du Québec à Trois-Rivières, 1990; Christine Payeur, Maternal Mortality in Quebec from the Medical Perspective and the Women's Point of View, 1890-1950, mémoire de mầtrise (histoire), Université de Montréal, 1998; Yolande Potvin, La femme et l'avortement à la fin du $X I X^{e}$ siècle : les points de vue d'un médecin québécois rigoriste et d'un médecin français jugé déviant, mémoire de maîtrise (études québécoises), Université du Québec à Trois-Rivières, 1991.

41. Ce point de vue a été défendu par F. Rousseau, op. cit.; F. Guérard, «L'État, l’Église et la santé au Québec de 1887 à 1939 », Cahiers d'histoire, 17,1-2 (printemps-automne 1997) : 76-94. 
nes de vaisseaux qui filtraient l'arrivée d'immigrants malades avant qu'ils débarquent dans les ports des villes, et peut-être de la vaccination ${ }^{42}$, elles ne prenaient vraiment de l'ampleur que durant certaines épidémies.

L'accentuation du processus d'urbanisation et d'industrialisation dans un contexte de laisser-faire entraîne la croissance de quartiers pauvres et insalubres dans les grands centres, situation qui va appeler à des mesures plus rigoureuses. Avec les découvertes de la bactériologie ${ }^{43}$, les médecins ont précisément en projet toute une gamme d'interventions qu'ils s'efforcent de faire adopter par les autorités urbaines. Petit à petit, ils y parviennent et les villes québécoises se dotent de services d'hygiène publique permanents et de mieux en mieux organisés. La série de réformes alors mises en branle participent du mouvement de l'hygiénisme international, tout en s'inscrivant dans le mouvement de réformes urbaines qui balaie l'Amérique du Nord et qui tend à confier la direction des services municipaux à des experts rémunérés plutôt qu'aux élus ${ }^{44}$.

L'étude de ces réformes a donné lieu à diverses interprétations. Les spécialistes de l'histoire des travailleurs ont peint un tableau très sombre en dénonçant l'insuffisance et la désuétude des mesures adoptées à Montréal, l'incurie de ses élites et le retard de la métropole sur d'autres grandes villes, en particulier sur Toronto. D'autres auteurs, pour Québec notamment, ont reconduit cette image d'une ville industrielle malsaine aux autorités municipales lentes à réagir ${ }^{45}$.

Les travaux récents ont modifié la perspective en montrant plutôt comment les promoteurs de l'hygiène sont parvenus à faire adopter leurs

42. Sur les quarantaines et la vaccination au XIX ${ }^{\mathrm{e}}$ siècle, voir Geoffrey Bilson, « Science, Technology and 100 Years of Canadian Quarantine », dans Richard A. Jarrell et A. E. Roos, dir., Critical Issues in the History of Canadian Science, Technology and Medicine (Thornhill, Ont., HSTC Publications, 1983), 89-100; Barbara Tunis, « Public Vaccination in Lower Canada 1815-1823 : Controversy and a Dilemna », HR, 9,1-2 (1982) : 264-278; Michael Farley, P. Keating et O. Keel, « La vaccination à Montréal dans la seconde moitié du $19^{\mathrm{e}}$ siècle : pratiques, obstacles et résistances », dans M. Fournier, Y. Gingras et O. Keel, dir., Sciences et médecine au Québec, perspectives socio-historiques (Montréal, Institut québécois de recherche sur la culture, 1987), 87-127.

43. Sur l'introduction de la bactériologie en territoire québécois, voir notamment, D. Goulet, Des miasmes aux germes : l'impact de la bactériologie sur la pratique médicale au Québec (18701930), thèse de doctorat (histoire), Université de Montréal, 1992; D. Goulet et O. Keel, « Les hommes-relais de la bactériologie en territoire québécois et l'introduction de nouvelles pratiques diagnostiques et thérapeutiques (1890-1920) », RHAF, 46,3 (hiver 1993) : 417-442.

44. Voir Raymond A. Mohl, The New City. Urban America in the Industrial Age, 1880-1920 (Illinois, Harlan Davidson, 1985).

45. Réjean Lemoine, « La santé publique : de l'inertie municipale à l'offensive hygiéniste », dans G.-Henri Dagneau, dir., La ville de Québec. Histoire municipale, 4 : De la Confédération à la charte de 1929 (Québec, La société historique de Québec, 1983), 153-180; Hervé Anctil et Marc-André Bluteau, La santé et l'assistance publique au Québec, 1886-1986 (Québec, Santé Société, édition spéciale, 1986). 
programmes par les groupes dirigeants. Déjà en 1982, des historiens des sciences avaient analysé les commencements, certes hésitants, du service de santé montréalais, en mettant l'accent sur l'inscription progressive de l'expertise médicale dans les processus décisionnels des autorités urbaines ${ }^{46}$. Dans une thèse de doctorat soutenue en 1996, Benoît Gaumer a retracé l'histoire de ce service, de sa mise sur pied en 1865 à son démantèlement en $1975^{47}$. Il estime que le service de santé montréalais connaît sensiblement les mêmes transformations que ceux des autres grandes villes américaines, que les nouveaux savoirs et pratiques y sont adoptés à un rythme comparable et qu'il convient donc de revenir sur le jugement sévère assené par Terry Copp. L'étude de Gaumer, on le voit, s'oppose aussi à la thèse d'un « retard culturel » du Québec.

Cette question d'un quelconque retard, démenti par les uns, ressuscité par les autres, se trouve souvent au centre des débats, bien que sa pertinence heuristique ait été contestée ${ }^{48}$. Il suffit pour s'en convaincre de consulter les travaux portant sur l'épidémie de variole de 1885 à Montréal. Michael Farley, Peter Keating et Othmar Keel ont argumenté que les résistances à la vaccination à Montréal dans la deuxième moitié du XIX ${ }^{\mathrm{e}}$ siècle, et notamment durant l'épidémie de 1885 , pouvaient être attribuées à bien d'autres causes que l'ignorance de la population canadienne-française et le passéisme de ses élites ${ }^{49}$. En intégrant la pratique de la vaccination dans le cadre plus large du processus de médicalisation de la société québécoise, ils ont révélé des obstacles d'ordre aussi bien scientifiques et techniques que politiques et socioculturels. Michael Bliss, dans un livre qui relève à la fois de l'enquête historique et, dans sa forme, du roman et du reportage, ramène l' analyse à un autre niveau en se montrant plus prompt à identifier des coupables : il réintroduit « la peur, l'ignorance et l'apathie » et insiste sur «l'erreur humaine » bien campée ici par

46. Ce texte a été republié récemment : M. Farley, O. Keel et Camille Limoges, « Les commencements de l'administration montréalaise de la santé publique (1865-1885) », dans P. Keating et O. Keel, dir., op. cit., 85-114.

47. Voir la thèse de B. Gaumer, op. cit.; B. Gaumer, G. Desrosiers, O. Keel et C. Déziel, « Le service de santé de Montréal : de l'établissement au démantèlement (1865-1975) », dans J.-P. Goubert et O. Keel, dir., op. cit., 131-158.

48. Dans le champ de l'histoire des sciences, voir l'introduction de M. Fournier, Y. Gingras et O. Keel, dir., op. cit. Divers auteurs qui ont travaillé sur l'enseignement de la médecine se sont également interrogés sur la lenteur des facultés de médecine francophones à adopter les normes américaines au $\mathrm{XX}^{\mathrm{e}}$ siècle et sur leur attachement au modèle français. Ils ont proposé des interprétations qui écartaient l'idée d'un « retard culturel » en privilégiant d'autres explications. Voir la série d'études déjà mentionnées en notes infrapaginales.

49. M. Farley, P. Keating et $\mathrm{O}$. Keel, «La vaccination à Montréal... », dans M. Fournier, Y. Gingras et O. Keel, dir., op. cit. 
un éminent médecin et homme politique d'ascendance irlandaise, ce à quoi il ajoute un zeste de «malchance ${ }^{50} »$.

De fortes réticences devant le processus de médicalisation ont également été relevées par les quelques chercheurs qui ont étudié l'hygiène publique dans les centres régionaux ${ }^{51}$. Dans ma thèse consacrée à la santé publique à Trois-Rivières et à Shawinigan de 1887 à 1939, j' ai mis au jour les rôles des divers acteurs dans les réformes sanitaires, de même que les appuis et les résistances aux projets des réformateurs. La plupart des acteurs sociaux, et cela vaut pour les médecins, le clergé et les hommes politiques de toutes tendances, sont apparus susceptibles de soutenir certaines mesures, tout en s'opposant à d'autres, dans un chassé-croisé d'alliances mouvantes.

Les édiles municipaux trifluviens et shawiniganais se sont montrés souvent opposés aux menées de l'organisme provincial d'hygiène qui n'a pu faire appliquer la Loi d'hygiène dans toute sa rigueur. Il est vrai que le processus de centralisation des décisions au sein de cet organisme représentait une perte de pouvoir pour les élites locales. Cette centralisation, également étudiée par Denis Goulet ${ }^{52}$, s'accompagne d'un effort de normalisation et culmine avec le remplacement graduel des services municipaux par des unités sanitaires. Les unités, véritables relais des programmes de l'organisme central, en viendront à desservir non seulement la population rurale de la province, mais aussi celle de la quasitotalité des villes du Québec. Leur implantation, ont souligné Georges Desrosiers, Benoît Gaumer et Othmar Keel, a été plus rapide et complète qu'ailleurs au Canada ou aux États-Unis, ce qui, estiment-ils, met à mal l'image « d'un gouvernement et d'une classe dirigeante [...] indifférents avant les années 1960 aux questions de santé publique ${ }^{53} »$.

50. Michael Bliss, Montréal au temps du grand fléau. L'histoire de l'épidémie de 1885 (Montréal, Libre Expression, 1993), 305-306. Voir aussi Donald C. Firth, A Tale of Two Cities : Montreal and the Smallpox Epidemic of 1885, mémoire de maîtrise, Université d'Ottawa, 1983.

51. Voir Julie Pomerleau, Comment appliquer les politiques provinciales d'hygiène : le cas des comtés de Brome et Missisquoi de 1870 à 1920, mémoire de maîtrise (histoire), Université de Sherbrooke, 1994; F. Guérard, La santé publique..., op. cit. Durant l'épidémie d'influenza de 1918, par contre, on observe une large mobilisation des élites : Denise Rioux, La grippe espagnole à Sherbrooke en 1918, mémoire de maîtrise (histoire), Université de Sherbrooke, 1985.

52. D. Goulet, «Des bureaux d'hygiène municipaux aux unités sanitaires. Le Conseil d'hygiène de la province de Québec et la structuration d'un système de santé publique, 1886-1926 », RHAF , 49,4 (printemps 1996) : 491-520; voir aussi F. Guérard, « L’hygiène publique au Québec de 1887 à 1939 : centralisation, normalisation et médicalisation », RS (mai-août 1996) : 203-227.

53. G. Desrosiers, B. Gaumer et O. Keel, La santé publique au Québec : histoire des unités sanitaires de comté : 1926-1975 (Montréal, Presses de l'Université de Montréal, 1998), 166. 
L'un des constats les plus fréquemment énoncés, et qui tranchait avec les interprétations antérieures, a été le rôle prépondérant de l'État ${ }^{54}$ dans l'établissement du système d'hygiène publique, sous l'impulsion de l'élite médicale du Québec. Quelques auteurs ont montré que l'État se faisait ainsi l'un des principaux agents de la médicalisation de la société québécoise et y assurait une uniformisation des pratiques sanitaires. De façon générale, on découvre petit à petit un État plus prompt à intervenir qu'on a pu le croire. Cela vaut pour le domaine hospitalier où les gouvernements du Québec, certes timidement puisqu'ils se gardent bien avant 1960 de s'ingérer dans le fonctionnement interne de l'hôpital, ont usé des fonds publics pour soutenir les établissements et promouvoir leur modernisation $^{55}$. La réflexion sur le rôle de l'État dans le champ de la santé demeure toutefois peu élaborée et gagnerait beaucoup à s'alimenter aux travaux récents entrepris dans d'autres champs de recherche ${ }^{56}$. Dans cette voie, on note tout de même un regain d'intérêt pour l'implantation de l'État-providence, notamment pour ses premières manifestations : pensions aux mères nécessiteuses, pensions de vieillesse, allocations familiales, assurance-chômage ${ }^{57}$.

En ce qui a trait aux hôpitaux, les nombreuses monographies publiées avant les années 1980 adoptent généralement une démarche où la commémoration de l'œuvre accomplie prend le pas sur toute autre considération. Peu sensibles aux influences extérieures qui contribuent à façonner l'hôpital, ces ouvrages tendent à le présenter comme un monde isolé et consensuel, évoluant en quelque sorte de son propre chef. Qu'il soit situé en milieu urbain n'est pas vraiment pris en considération. Toutefois, nous disposons maintenant de monographies qui font de l'hôpital une institution éminemment urbaine dans ses rapports à la population locale et régionale et aux autorités publiques. Les livres de Normand Perron, de François Rousseau, de Denis Goulet, de François Hudon et d'Othmar Keel, ma thèse et celle de François Hudon sont autant de textes qui, à dif-

54. C'était un des points forts de l'étude de H. Anctil et de M.-A. Bluteau, op. cit.

55. F. Guérard, La santé publique..., op. cit.; et id., Histoire de la santé..., op. cit.

56. Certains déjà sont tentés par pareille démarche : Walter Sendzik, The 1832 Montreal Cholera Epidemic : A Study in State Formation, mémoire de maîtrise (histoire), Université McGill, 1997.

57. Sur ces questions, voir par exemple, Yves Vaillancourt, L'évolution des politiques sociales au Québec, 1940-1960 (Montréal, Presses de l'Université de Montréal, 1988); Dennis Guest, Histoire de la sécurité sociale au Canada (Montréal, Boréal, 1993); Dominique Marshall, Aux origines sociales de l'État-providence : familles québécoises, obligation scolaire et allocations familiales, 1940-1955 (Montréal, Presses de l'Université de Montréal, 1998). Dans une recherche en cours, A. Charles aborde les pensions de vieillesse. 
férents degrés, mettent en relief l'imbrication de l'hôpital à son environnement social, politique et économique ${ }^{58}$.

Ces études de cas ont mis en lumière le processus de médicalisation qui métamorphose l'hôpital et l'autorité grandissante des médecins sur l'organisation des services. Ce processus progresse inégalement sur le territoire ou selon le type d'hôpitaux. Ainsi, dans les établissements pour malades mentaux, étudiés par plusieurs auteurs ${ }^{59}$, il semble que la montée de l'influence des médecins s'effectue plus lentement ou avec moins d'intensité que dans les hôpitaux généraux, exception faite des hôpitaux psychiatriques anglophones.

Globalement, on y voit déjà plus clair dans les rapports parfois conflictuels qui s'établissent entre médecins, communautés religieuses, philanthropes, entreprises, municipalités, clientèle et État, et on perçoit

58. Normand Perron, Un siècle de vie hospitalière au Québec. Les Augustines et l'Hôtel-Dieu de Chicoutimi, 1884-1984 (Québec, Presses de l'Université du Québec, 1984); F. Rousseau, La croix et le scalpel. Histoire des Augustines et de l'Hôtel-Dieu de Québec, 1 : 1639-1892 et 2 : 1892-1989 (Sillery, Septentrion, 1989 et 1994); F. Guérard, La santé publique..., op. cit.; id., « Les principaux intervenants dans l'évolution du système hospitalier en Mauricie, 1889-1939 », RHAF, 48,3 (hiver 1995) : 375-401; D. Goulet, François Hudon et O. Keel, Histoire de l'Hôpital Notre-Dame de Montréal, 1880-1980 (Montréal, vlb éditeur, coll. «Études québécoises », 1993); F. Hudon, L’hôpital comme microcosme de la société : enjeux institutionnels et besoins sociaux à l'Hôpital Notre-Dame de Montréal, 1880-1960, thèse de doctorat (histoire), Université de Montréal, 1996. Voir aussi Danielle Choquette, Histoire de l'Institut de réadaptation de Montréal de 1949 à 1976, mémoire de maîtrise (histoire), Université de Montréal, 1996; Rita Desjardins, Les débuts de l'Hôpital SainteJustine à Montréal, 1907-1921, mémoire de maîtrise (histoire), Université de Montréal, 1989; MarieJosée Fleury, L'Hôpital Saint-Paul (1905-1934) et sa contribution à la prévention et à la lutte contre les maladies contagieuses, mémoire de maîtrise (histoire), Université de Montréal, 1993; Laurent Rodrigue, Fondation et développement d'un hôpital laïc [sic]sous contrôle médical : l'Hôtel-Dieu de Sherbrooke (1917-1943), mémoire de maîtrise (histoire), Université de Sherbrooke, 1994.

59. Une vaste littérature existe sur l'histoire de la santé mentale au Québec : Céline Beaudet, Évolution de la psychiatrie anglophone au Québec, 1880-1963 : le cas de l'Hôpital de Verdun (Québec, Université Laval, Cahiers de l'Institut supérieur des sciences humaines, coll, «Études sur le Québec », $\mathrm{n}^{\circ}$ 6, 1976); Guy Boisclair, La perception de la folie au Québec au XVIII siècle, mémoire de maîtrise (histoire), Université de Sherbrooke, 1989; Françoise Boudreau, De l'asile à la santé mentale. Les soins psychiatriques : histoire et institutions (Montréal, Éditions Albert Saint-Martin, 1984); Daniel Bouchard et Simon Doucet, L'État et l'administration des institutions asilaires au Québec : 1845-1895, mémoire de maîtrise (histoire), Université du Québec à Montréal, 1986; André Cellard, Histoire de la folie au Québec de 1600 à 1850. Le désordre (Montréal, Boréal, 1991); A. Cellard et D. Nadon, « Ordre et désordre : le Montreal Lunatic Asylum et la naissance de l'asile au Québec », RHAF, 39,3 (hiver 1986) : 345-369; Guy Grenier, « Doctrine de la dégénérescence et institution asilaire au Québec (1885-1930) », dans J.-P. Goubert et O. Keel, dir., op. cit., 105-116; id., L'histoire de la folie criminelle au Québec de 1840 à 1945, thèse de doctorat, Université de Montréal, 1998; P. Keating, La science du mal : l'institution de la psychiatrie au Québec : 1800-1914 (Montréal, Boréal, 1993); A. Paradis, «L'asile québécois et les obstacles à la médicalisation de la folie », CBMH/BCHM, 11,2 (1994) : 297-334; id., «L'asile, de 1845 à 1920 », dans N. Séguin, dir., op. cit., 37-73; id., « Thomas J. W. Burgess et l'administration du Verdun Protestant Hospital for the Insane (1890-1916) », CBMH/BCHM, 14,1 (1997) : 5-35; id., « Le sousfinancement gouvernemental et son impact sur le développement des asiles francophones au Québec (1845-1918) », RHAF, 50,4 (printemps 1997) : 571-598; A. Paradis et al., " L'émergence de l'asile québécois au XIX ${ }^{\mathrm{e}}$ siècle », Santé mentale au Québec, 2,2 (1977) : 1-44. 
mieux leurs rôles respectifs dans les transformations qui ont marqué l'hôpital de la fin du $\mathrm{XIX}^{\mathrm{e}}$ siècle et de la première moitié du $\mathrm{XX}^{\mathrm{e}}$. Là encore, les chercheurs ont levé le voile sur une diversité insoupçonnée, par exemple en ce qui a trait à la propriété et à la gestion de l'hôpital, à son financement et aux caractéristiques du personnel.

Malgré cette progression des connaissances sur l'histoire des hôpitaux, il reste beaucoup à faire avant que se dégage une vision globale du développement du dispositif hospitalier, dont on peut tout de même trouver une amorce dans le dernier tome de l'Atlas historique du Québec ${ }^{60}$. Ainsi, alors que l'on dispose de bonnes monographies d'hôpitaux généraux, les hôpitaux spécialisés sont restés dans l'ombre, à l'exception des établissements pour malades mentaux. Encore plus étonnant, dans une société où les communautés religieuses ont pris en charge une majorité d'hôpitaux et sont ainsi devenues propriétaires ou gestionnaires de biens immobiliers considérables, ces communautés n'ont pas encore été étudiées spécifiquement en tant qu'organisations dotées de leur propre logique de développement, c'est-à-dire un peu à la façon de l'histoire des entreprises. Certains services sociaux ${ }^{61}$, comme les hospices ${ }^{62}$, mériteraient plus d'attention, ne serait-ce que parce que leur développement offre beaucoup de similitudes avec celui des hôpitaux et participe bien souvent d'un même mouvement, bien qu'ils n'aient pas connu avec une intensité comparable le processus de médicalisation ${ }^{63}$. Il faudrait aussi revenir sur la question des assurances pour l'hospitalisation : sociétés mutuelles, ententes entre les hôpitaux et les entreprises pour leurs employés, application de la loi sur les accidents du travail et rôle de l'État, assurances privées après la Seconde Guerre mondiale. Enfin, les transformations du personnel hospitalier n'ont pas été suffisamment étudiées, quoique la thèse de doctorat d'Aline Charles sur le travail et la vieillesse

60. N. Séguin, dir., op. cit.

61. Sur les services sociaux, certains travaux récents laissent entrevoir des développements prometteu'rs. Voir, par exemple, France Gagnon, L'hospice Saint-Joseph de la Maternité de Québec, 1852-1876 : prise en charge de la maternité hors-norme (Québec, Groupe de recherche multidisciplinaire féministe, Université Laval, coll. « Cahiers de recherche du GREMF », nº 70, 1996).

62. Voir Bettina Bradbury, « Mourir chrétiennement. La vie et la mort dans les établissements catholiques pour personnes âgées à Montréal au XIX e siècle », RHAF, 46,1 (été 1992) : 143-175.

63. Ajoutons tout de même que de nombreux travaux traitent des programmes étatiques pour venir en aide aux indigents ou aux institutions qui les hébergent. 
féminine en milieu hospitalier de 1940 à 1980 ait ouvert là-dessus des perspectives novatrices ${ }^{64}$.

Par ailleurs, si les processus de professionnalisation et de médicalisation ont beaucoup retenu l'attention, on n'est pas encore vraiment parvenu à prendre le pouls de la population ${ }^{65}$, des malades et des patients ${ }^{66}$, même si diverses résistances ou appuis aux réformes ont été mis au jour. L'évolution de leurs perceptions a certainement, par moments, constitué un frein ou préparé le terrain à divers changements, que ce soit dans l'univers hospitalier ou dans celui de l'hygiène publique. Nul doute qu'une étude sur les clientèles d'hôpitaux, par exemple, apporterait des éclairages révélateurs.

\section{L'ÉVOLUTION DE L'ÉTAT DE SANTÉ DES POPULATIONS}

Une autre question a surgi à l'examen des services de santé : quelle influence ont-ils exercée sur l'état de santé des populations urbaines? Les recherches d'historiens et de démographes ${ }^{67}$ font penser que pour les $\mathrm{XVII}^{\mathrm{e}}$ et $\mathrm{XVIII}^{\mathrm{e}}$ siècles, et même pour le $\mathrm{XIX}^{\mathrm{e}}$, les mesures adoptées n'ont pas sensiblement infléchi la mortalité. Parmi les études de démographes, celle de Danielle Gauvreau sur la population de la ville de Québec au temps de la Nouvelle-France mérite d'être soulignée ${ }^{68}$. Dans une démarche rigoureuse, elle confirme notamment certains traits démographiques déjà soulignés par d'autres auteurs ${ }^{69}$ : une mortalité générale marquée par des crises au gré des épidémies; une mortalité plus forte au

64. A. Charles, Travail et vieillesses féminines..., op. cit. Voir aussi Yves Belzile, Syndicalisme et conditions de travail chez les employés de l'Hôpital Notre-Dame de Montréal (1935-1980), mémoire de maîtrise (histoire), Université de Montréal, 1992; J. Daigle, «La syndicalisation des infirmières », dans M. Lavigne, et al., dir., op. cit.; id., « Une "révolution" dans la tradition : les réformes dans le champ de la santé au cours des années 1960 et l'organisation du travail hospitalier », dans R. Comeau, dir., op. cit., 148-154; André Valiquette, L'essor du syndicalisme catholique chez. les employé(s) d'hôpitaux du Québec dans les années trente et quarante, mémoire de maîtrise (histoire), Université du Québec à Montréal, 1982.

65. À ce propos, voir B. Bradbury, « The Fragmented Family : Family Strategies in the Face of Death, Illness and Poverty, Montreal, 1860-1885 », dans Joy Parr, dir., Childhood and Family in Canadian History (Toronto, McClelland \& Stewart, 1982), 109-128.

66. Pour des études centrées sur les patients, voir A. Lévesque, La santé des femmes en période de dépression économique : l'exemple des patientes de l'Hôpital de la Miséricorde à Montréal pendant les années trente (Montréal, Bulletin du regroupement des chercheurs-chercheures en histoire des travailleurs et travailleuses du Québec, 1990); Louise Côté, "En garde! » : les représentations de la tuberculose au Québec dans la première moitié $d u X X^{e}$ siècle : maladie, culture et identité, thèse de doctorat (histoire), Université Laval, 1997.

67. Si les travaux de démographie historique qui traitent de la mortalité ne portent généralement pas spécifiquement sur le milieu urbain, plusieurs apportent des informations fort utiles.

68. Voir D. Gauvreau, Québec. Une ville et sa population au temps de la Nouvelle-France (Québec, Presses de l'Université du Québec, 1991).

69. À ce sujet, voir par exemple, Lorraine Gadoury, Yves Landry et Hubert Charbonneau, «Démographie différentielle en Nouvelle-France : villes et campagnes », RHAF, 38,3 (hiver 1985) : 357-378. 
XVIII siècle qu'au XVII ${ }^{\mathrm{e}}$ et à Québec qu'en milieu rural. Notons que l'écart entre les mortalités urbaine et rurale ne sera pleinement résorbé que bien plus tard, vers 1940. Danielle Gauvreau établit également que les risques de décéder avant l'âge d'un an sont nettement plus élevés dans les catégories socioprofessionnelles dont les conditions de vie sont les moins avantageuses, constat que l'on peut reporter jusqu'à aujourd'hui.

Les hauts niveaux de mortalité se maintiennent jusqu'au $\mathrm{XX}^{\mathrm{e}}$ siècle. Jean-Claude Robert, par exemple, a montré que l'industrialisation de Montréal au XIX ${ }^{\mathrm{e}}$ siècle n'a pas modifié substantiellement, que ce soit à la hausse ou à la baisse, une mortalité exceptionnellement élevée ${ }^{70}$. En fait, le recul accéléré de la mortalité dans les villes débute après la mise en place généralisée de services d'hygiène publique stables. Aussi paraît-il tentant de leur en attribuer la paternité. Pour Montréal, Benoît Gaumer a cherché à préciser l'effet des mesures entreprises pour lutter contre certaines causes de décès ${ }^{71}$. Il estime que certaines de ces mesures ont sans doute contribué à la réduction de la mortalité. J'ai établi qu'il en va de même à TroisRivières, tout particulièrement dans le cas de l'assainissement de l'eau ${ }^{72}$. Nous avons toutefois dû reconnaître que l'action conjuguée d'autres facteurs interdit de préciser vraiment les effets des interventions sanitaires et ce, d'autant plus que la morbidité demeure insaisissable ${ }^{73}$.

De façon générale, l'étude de l'évolution de l'état de santé des populations citadines au Québec, particulièrement avant 192674, repose encore sur des bases fragiles. Des collaborations entre historiens, démographes et épidémiologues permettraient sans doute d'obtenir des séries mieux contrôlées et, surtout, de fixer des balises méthodologiques qui tiennent vraiment compte des spécificités de nos sources statistiques. Alors seulement les comparaisons avec d'autres lieux deviendront moins sujettes à caution. Autre tâche ardue mais essentielle, il faudrait mieux situer dans la diachronie les transformations des déterminants de la santé autres que les services de santé. Les

70. J.-C. Robert, «The City of Wealth and Death... », dans W. Mitchinson et J. Dickin McGinnis, dir., op. cit.

71. Voir la thèse de B. Gaumer, op. cit.

72. F. Guérard, La santé publique..., op. cit.

73. Une étude récente a rencontré des difficultés similaires : Catherine Braithwaite, P. Keating et Sandi Viger, «The Problem of Diphteria in the Province of Quebec : 1894-1909 », HS/SH, 29,57 (mai 1996) : 71-95.

74. En 1926, à la suite d'une entente entre les gouvernements fédéral et provincial, la collecte des données démographiques et leur traitement sont organisés sur des bases plus rigoureuses. 
modes de vie, l'alimentation ${ }^{75}$, l'hygiène personnelle et les habitudes vis-à-vis de la consommation des services, les pratiques populaires ${ }^{76}$ demeurent mal connus.

\section{LE RÔLE CENTRAL DES PÔLES URBAINS}

Dans une perspective géographique cette fois, à l'échelle du Québec, s'impose une partition du territoire en deux grandes aires d'influence, l'Est et l'Ouest, articulées aux pôles urbains de Québec et de Montréal. Elles se rencontrent et se chevauchent le long d'une bande qui traverse le lac Saint-Pierre, et sont donc le décalque exact des aires d'influence socio-économiques de la capitale et de la métropole. Qu'il s'agisse de la provenance des étudiants de médecine et des lieux de pratique des diplômés, comme l'a montré George Weisz ${ }^{77}$, des clientèles des asiles de Beauport et Saint-Jean-de-Dieu, tel que souligné par André Paradis ${ }^{78}$, des affiliations universitaires des écoles d'infirmières et de la clientèle extérieure des hôpitaux, dont j'ai traité ${ }^{79}$, deux blocs se détachent, particulièrement aux $\mathrm{XIX}^{\mathrm{e}}$ et $\mathrm{XX}^{\mathrm{e}}$ siècles. Ils témoignent à l'évidence du rôle central des deux seules agglomérations majeures.

À plus d'un titre, la hiérarchie des pôles urbains se fait sentir dans l'organisation des services et des groupes professionnels. Les services spécialisés qui recourent à une technologie avancée et à un personnel hautement qualifié sont concentrés à Montréal et à Québec, là où logent les grandes facultés de médecine. L'élite médicale de ces deux villes définit les grands projets de réforme et encadre leur mise en œuvre. Elle exerce une large influence à travers les organismes gouvernementaux, préside aux destinées de la profession par le truchement du Collège des médecins et chirurgiens, dirige les publications médicales. Cette hégémo-

75. Voir à ce sujet F. Rousseau, L'œuvre de chère en Nouvelle-France. Le régime des malades à l'Hôtel-Dieu de Québec (Québec, Les Presses de l'Université Laval, 1983); W. Peter Ward et Patricia C. Ward, «Infant Birth Weight and Nutrition in Industrializing Montreal », AHR, 89 (1984) : $324-$ 345; Donald William Fyson, Eating in the City : Diet and Provisioning in Early Nineteenth-Century Montreal, mémoire de maîtrise (histoire), Université McGill, 1989.

76. Pour une présentation des principaux travaux sur la médecine populaire, voir F. Saillant, et al., Se soigner en famille : les recettes de médecine québécoise dans les familles québécoises du début du XXe siècle (Centre de recherche sur les services communautaires, Université Laval, 1990). Voir aussi $i d$., «Savoirs et pratiques des femmes dans l'univers ethnomédical québécois », Folklore canadien/Canadian Folklore, 14,1 (1992) : 47-72; D. Goulet, Le commerce des maladies. La publicité des remèdes au début du siècle (Québec, Institut québécois de recherche sur la culture, 1987); Guildo Rousseau, «La santé par correspondance : un mode de mise en marché des médicaments brevetés au début du siècle », HS/SH, 28,55 (mai 1995) : 1-25.

77. G. Weisz, « Origines géographiques et... », dans M. Fournier, Y. Gingras et O. Keel, dir., op. cit.

78. A. Paradis, « L'asile, de 1845 à $1920 »$, dans N. Séguin, dir., op. cit., 37-73.

79. F. Guérard, «Les grands appareils sanitaires », dans N. Séguin, dir., op. cit. 
nie n'est pas sans susciter un mécontentement parmi les médecins du reste du Québec qui estiment parfois leurs intérêts lésés ${ }^{80}$.

À une autre échelle, on commence, par bribes, à percevoir l'influence des centres régionaux sur leur arrière-pays, notamment en ce qui a trait à la médicalisation vraisemblablement plus lente du milieu rural. Ce peut être à titre de relais des programmes définis à Montréal et à Québec, comme dans le cas des unités sanitaires de comté qui, situées dans de petites villes, desservent les campagnes environnantes. Ce peut être aussi à titre de centres autonomes de services régionaux, particulièrement de services sociaux et hospitaliers. Une étude approfondie des clientèles hospitalières, encore une fois, livrerait des informations originales sur cette question peu abordée. L'influence des petites villes peut également emprunter d'autres voies : à travers les sociétés médicales régionales par exemple, ou par l'imposition, parfois controversée comme cela a été démontré pour Trois-Rivières, d'une réglementation sanitaire affectant la production et l'échange des aliments ${ }^{81}$.

Notre compréhension du rôle des centres urbains dans la diffusion des savoirs et des pratiques demeure toutefois bien partielle, notamment en ce qui a trait à l'effacement graduel d'une médecine populaire enracinée de longue date et à ses transformations. Il faut sans doute en chercher la cause dans la rareté des études historiques consacrées à la santé en milieu rural.

\section{CONCLUSION}

Les derniers vingt ans de recherche historienne sur la médecine et la santé ont bouleversé les perceptions de notre passé sanitaire. En inscrivant leurs travaux dans le courant de l'histoire sociale, en privilégiant les interactions entre groupes sociaux et leurs rapports à la santé, plutôt que la célébration de l'élite médicale et des innovations scientifiques, en recourant à des mises en contexte larges qui intègrent, outre l'état des connaissances et de la pratique médicales, les dimensions économiques, politiques, sociales et culturelles, les historiens ont conféré à ce champ de recherche une ample portée. C'est, pour certains, à l'histoire de la société québécoise à travers le prisme de la santé qu'ils se sont livrés, dans une démarche qui participe aux grands cheminements de l'historiographie du Québec.

80. D. Goulet, Histoire du Collège des médecins..., op. cit.; id, « La structuration de la pratique médicale, 1800-1940 », dans N. Séguin, dir., op. cit., 117-153.

81. F. Guérard, La santé publique..., op. cit. 
L'examen de l'état de santé de la population a éclairé l'évolution des conditions de vie et enrichi l'histoire des travailleurs. Celui de l'implantation des services a contribué au développement des études urbaines, entre autres en permettant d'appréhender les processus décisionnels au sein des organismes publics et privés et en faisant ressortir l'influence montante des experts, de même que diverses résistances aux réformes. D'intéressants appendices aux discours des élites ont été mis au jour, qui affinent nos connaissances sur les idéologies au Québec. Les différences, dans bien des cas les différends, mais aussi les collaborations entre les principaux groupes ethniques et linguistiques ont été explorées. À ceux qu'intéressent les grandes institutions, qui s'interrogent sur l'État québécois ou sur l'Église, sur leurs rôles respectifs et sur le partage de leurs territoires d'intervention, l'histoire de la santé a livré des matériaux rafraîchissants. En outre, la santé est devenue un champ de prédilection pour l'histoire des femmes, que ce soit à titre d'actrices, souvent de premier plan, dans l'organisation des services, ou comme cibles des campagnes sanitaires.

Dans un premier temps, il importait d'ancrer fermement les repères essentiels de la mise en place et de l'organisation des services de santé en exploitant la documentation très riche dont regorgent les archives gouvernementales, municipales et hospitalières. Dans l'ensemble, cela a été fait. Il reste, certes, des zones d'ombre. Certaines ont été mentionnées. Ajoutons-y que la recherche historienne sur la santé dans la ville s'est peu aventurée dans la période 1945-1960, et exceptionnellement au-delà de 1960. Il faudra bien pourtant en venir à faire le pont entre nos travaux et ceux des chercheurs d'autres disciplines sur les quarante dernières années. L'ajustement risque de s'avérer parfois difficile, dans la mesure où les interprétations issues des nouveaux travaux historiens bousculent des perceptions jusqu'alors assez communément admises sur notre passé sanitaire, perceptions dans certains cas stéréotypées, étriquées même - on pense ici notamment aux clichés de la domination sans partage du clergé et de l'inertie de l'État - puisque construites en l'absence d'un corpus de recherches historiques rigoureusement menées. Ce corpus a maintenant pris forme. Étendons son emprise quelques décennies plus avant, si nous voulons que l'histoire nourrisse les débats actuels sur l'évolution du système de santé. Certains chercheurs ont déjà emprunté cette voie.

Concurremment à l'étude de la mise en place et de l'organisation des services, la nature des rapports qu'entretenaient les différents acteurs, les enjeux que représentaient pour eux les transformations du monde sanitaire, ont été explorés. C'est sans doute là qu'il faut situer les acquis les plus importants. En mettant en lumière une remarquable 
diversité d'opinions entre les groupes et même en leur sein, en levant le rideau sur des élites ouvertes aux courants d'idées internationaux, beaucoup moins rétives à la modernité qu'on l'avait cru, l'histoire de la santé a apporté une contribution notable au renouvellement de l'historiographie québécoise. Il est ainsi apparu, dans le domaine sanitaire, que le traditionalisme de certains pouvait se marier sans trop de remaniements avec une volonté clairement exprimée de modernisation. Ce traditionalisme n'a donc pas constitué un frein aussi puissant qu'on a pu le dire.

Forte de tels acquis, l'histoire de la santé doit maintenant éviter de s'enferrer dans des débats bientôt stériles, si ce n'est déjà le cas. S'interroger encore et encore sur les « retards » éventuels du Québec et sur leurs causes a nettement perdu de sa pertinence. Les comparaisons avec l'ailleurs ont d'autres finalités que d'encenser des supériorités ou de stigmatiser de présumées infériorités. Elles doivent servir à identifier ce qui est commun et ce qui est spécifique, les grands courants et les adaptations locales, et à expliquer ces dernières dans des mises en contexte larges.

Ce bilan de la recherche amène aussi un constat un peu surprenant : celui de la rareté des débats de fond. Les historiens ont, certes, récusé l'optique qui dominait l'histoire traditionnelle de la médecine. Plusieurs se sont opposés à l'idée d'un retard culturel, naguère si prégnante, ou se sont démarqués des travaux des années 1970 en livrant une image moins sombre de la santé publique au Québec. Mais, curieusement, les publications font peu état de divergences entre les spécialistes actuels, sinon, parfois, dans des comptes rendus de livres ${ }^{82}$. Il est vrai que tous, ou presque, se réclament de l'histoire sociale et partagent les mêmes paradigmes scientifiques, en particulier la nécessité de situer l'objet d'étude dans son contexte social, économique et politique. La pratique de l'histoire de la santé au Québec, les approches et les interprétations n'en sont pas pour autant uniformes. Par exemple, le poids accordé aux innovations médicales comme facteurs de changement et comme éléments structurants de l'organisation des services de santé diffère nettement d'un auteur à l'autre. Un dialogue plus soutenu entre les spécialistes et l'éventuelle confrontation de leurs idées paraissent maintenant nécessaires à un approfondissement de la réflexion et à une clarification des enjeux de la recherche.

82. Nous avons relevé les comptes rendus effectués depuis dix ans dans la Revue d'histoire de l'Amérique française, Recherches sociographiques, Histoire sociale/Social History et le Canadian Bulletin of Medical History/Bulletin canadien d'histoire de la médecine. 
Enfin, il serait bon de prendre un certain recul et, en mettant notamment à profit les monographies disponibles, d'embrasser des terrains d'enquête élargis afin de dégager des vues d'ensemble. Il ne s'agit pas ici, bien au contraire, d'abandonner les études de cas et la diversification des thèmes : elles demeurent indispensables à la profondeur de vue, ne seraitce qu'en interdisant les généralisations bâclées. Mais cette parcellarisation de la recherche doit jeter les fondements de mises en forme plus vastes. On peut souhaiter que la moisson de connaissances nouvelles dont j'ai fait état, un meilleur arrimage aussi aux acquis d'autres champs de recherche, fourniront les matériaux de schémas explicatifs amples fondés sur une compréhension de la complexité sous-jacente. 